\title{
ROBÓTICA COMO METODOLOGIA DE APOIO À DISCIPLINA DE INTRODUÇÃO À ENGENHARIA
}

DOI: 10.37702/2175-957X.COBENGE.2021.3509

Débora Porto Barbosa - deburith@yahoo.com.br

Instituto de Metrologia e Qualidade do Estado de Minas Gerais IPEM MG

Rua Luiz Carlos Ribeiro 215

30642-300 - Belo Horizonte - MG

Marcos Maciel Peres - marcosmperes@gmail.com

PUC Minas

Rua Gilberto Corrêa e Castro 54

30411-530 - Belo Horizonte - MG

Marco Aurelio de Souza Birchal - birchal@pucminas.br

PUC Minas Pontifícia Universidade Católica de Minas Gerais

rua padre pedro evangelista 274

30535-490 - Belo Horizonte - MG

Resumo: Apresenta-se uma metodologia de apoio ao ensino, a ser empregada em disciplinas de Introdução à Engenharia e afins, que se utiliza de elementos de robótica e propõe o desenvolvimento tanto de um protótipo físico quanto virtual. Aproveita o conceito de gêmeos virtuais para introduzir o aluno à engenharia, buscando, de acordo com as atuais DCNs (Diretrizes Curriculares Nacionais) do Curso de Graduação em Engenharia, desenvolver competências esperadas de um engenheiro, tanto de soft skills quanto de hard skills. Para tanto, propõe, através de uma série de atividades de aprendizagem, explorar aspectos teóricos e práticos da construção de um protótipo robótico e de seu similar virtual, com auxilio de softwares livres.

Palavras-chave: Metodologia. Robótica. Introdução à Engenharia. TBL. 


\section{COBENGE \\ ROBÓTICA COMO METODOLOGIA DE APOIO À DISCIPLINA DE INTRODUÇÃO À ENGENHARIA}

\section{INTRODUÇÃO}

Desde meados da década de 1960 Seymour Papert, um dos fundadores do laboratório de Inteligência Artificial do Massachusetts Institute of Technology (MIT), baseado nas ideias de Jean Piaget, já pensava na introdução dos computadores nas escolas como forma de adaptar as tecnologias da época à metodologia de ensino (PIROLA, 2010). Seguindo o raciocínio de Papert e com o aparecimento de novas tecnologias, têm se tornado viável, para além da computação, também a robótica, como ferramenta de aprendizado.

A robótica é parte da solução moderna para a necessidade de se encontrar meios mais eficientes e mais seguros de produção, que possam não somente aumentar o rendimento do sistema produtivo, mas que também livrem o homem de tarefas repetitivas, insalubres ou perigosas.

Pensando no rápido desenvolvimento das tecnologias e no contato precoce das novas gerações com estas, é preciso encontrar métodos cada vez mais ágeis e interativos, que contribuam para instigar o interesse das pessoas aos campos da ciência e da tecnologia.

A robótica, como ferramenta pedagógica, ganha, portanto, força, por se tratar de uma aplicação de conceitos abstratos ao mundo físico, com o objetivo de disponibilizar aos alunos a oportunidade de criarem soluções voltadas ao mundo real, incorporando o raciocínio, lógica e cálculos matemáticos. Com a robótica, o aprendizado ocorre de forma dinâmica e estimulante, favorecendo o crescimento intelectual pela experimentação, construção e observação.

Este trabalho traz uma metodologia de ensino para a disciplina de Introdução à Engenharia, ou correlatas, que emprega ferramentas de software e técnicas de prototipação, de forma simplificada, porém com forte apelo educacional. Considera-se a multidisciplinaridade e o desenvolvimento de projetos aplicados, de forma a promover uma introdução consistente e estimulante, aos grandes temas da Engenharia.

Buscando maior interesse dos alunos, a dissociação entre o físico e o virtual flexibiliza o entendimento, permitindo a interação primária virtualmente, dando confiança para posterior aplicação das alterações reais. Neste sentido, a metodologia presente se utiliza do conceito de Gêmeos Digitais, para promover este paralelo entre o físico e virtual.

Segundo Cristina De Luca, Gêmeos Digitais (Digital Twins) consistem, basicamente, em se criar uma réplica digital de algo que se deseja ou com o que se precisa interagir ou compreender, podendo ser realizadas simulações, que facilitam a compreensão ou previsão de um resultado sob determinada mudança ou ação (LUCA, 2019).

Para que se alcance o objetivo descrito, esta metodologia se utiliza de recursos comuns, presentes ou disponíveis para a maioria das escolas de engenharia, tendo-se optado por ferramentas abertas de hardware e de software. Notadamente, adotaram-se a plataforma Arduino (ARDUINO, 2021) e o programa de simulação robótica CoppeliaSim (COPPELIA, 2021). 


\section{METODOLOGIA DO PROJETO}

A metodologia desenvolvida prevê o desenvolvimento de três fases, subdivididas em etapas. Cada etapa tem como foco uma ou mais habilidades a serem apreendidas.

A tabela 1 traz um detalhamento das fases e suas etapas.

\begin{tabular}{c|l}
\multicolumn{2}{c}{ Tabela 1 - Fases e suas etapas. } \\
\hline FASE & \multicolumn{1}{c}{ ETAPA } \\
\hline I -Introdução & 1- Apresentação do Problema \\
& 2 - Interação com o modelo Físico \\
& $3-$ Interação com o modelo virtual \\
\hline II - Física & $4-$ Reconhecimento dos componentes \\
& 5 - Montagem mecânica \\
& 6 - Montagem eletro/eletrônica \\
& 7 - Testes preliminares do protótipo \\
\hline III - Virtual & 8- Reconhecimento da plataforma virtual \\
& 9 - Construção do modelo virtual \\
& $10-$ Programação da interatividade \\
\hline
\end{tabular}

Fonte: Os autores

$\mathrm{Na}$ primeira fase, Introdução, ocorre a apresentação do problema aos alunos e um consequente primeiro contato com o projeto que terão que desenvolver. Esta fase se faz necessária já que, para muitos, este é o primeiro contato com um projeto de engenharia e se torna uma oportunidade para o aluno vivenciar as tecnologias de projeto, ainda como usuário.

A fase Física traz o primeiro contato do estudante com o projeto propriamente dito. Nela, habilidades mecânicas e eletro/eletrônicas serão exploradas.

A última fase, a Virtual, é aquela em que o aluno vivencia o desenvolvimento de um protótipo computacional, o projeto virtual do modelo físico que foi implementado na fase anterior. As habilidades aqui exploradas são, principalmente, aquelas relativas à computação aplicada à engenharia.

Assim posta, a metodologia apresentada vai ao encontro das expectativas das DCNs - Diretrizes Curriculares Nacionais do Curso de Graduação em Engenharia (MINISTÉRIO DA EDUCAÇÃO, 2019) - no sentido de criar um conjunto de atividades teóricas e práticas que explorem habilidades presentes em diferentes dimensões da engenharia, formando novas competências ou fortalecendo aquelas já existentes. Cada uma das fases e as habilidades pretendidas, serão, a seguir, discutidas.

Para que haja um maior engajamento do estudante, optou-se por implementar a metodologia segundo a estratégia TBL (Team Based Learning), o que reforça as competências relativas ao trabalho em grupo e a socialização entre os integrantes do grupo (CORDON e NETO, 2020).

\section{FASE - INTRODUÇÃO (I)}

Nesta fase o aluno entra em contato com um projeto já finalizado, na posição de um usuário power user, aos moldes daquele que ele também irá desenvolver. Ela se faz necessária para que o estudante possa tomar conhecimento das tecnologias envolvidas, dispositivos utilizados e possíveis soluções.

Os alunos são convidados a interagirem com os gêmeos digitais e a explorarem seus componentes, tanto físicos quanto lógicos, de forma a tentarem compreender o funcionamento do todo integrado. 
O protótipo físico proposto e desenvolvido neste trabalho se trata de uma plataforma recorrente em vários cursos introdutórios de robótica, qual seja, um carro robótico dotado de duas rodas ativas e independentes e um apoio. $\mathrm{O}$ carro é controlado por uma plataforma Arduino, também muito comum nos cursos de engenharia. Este carro robô deve ser clonado em seu gêmeo digital, através do software CoppeliaSim, que será capaz de comandar o par físico. A Figura 1 ilustra um carro típico.

Figura 1 - Carro robótico típico utilizado no projeto.

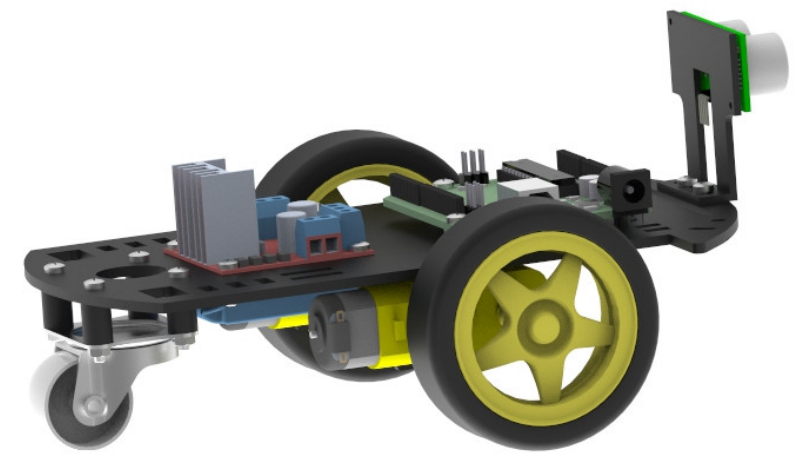

Fonte: https://grabcad.com/library/arduino-2wd-robot-chassis-1

O projeto a ser desenvolvido é apresentado ao aluno e cada uma de suas fases, explicada. Podem-se formar grupos e, ao final desta fase, um cronograma deve ser formulado por cada um destes grupos.

Daqui o que se espera é que haja um despertar consciente para novas habilidades e competências a serem exploradas ao longo do curso de engenharia.

\section{FASE FÍSICA (II)}

A fase Física é aquela em que o aluno tem contato direto com o hardware, os componentes, ou partes físicas do sistema a ser construído.

Esta Fase possui quatro Etapas: 4 - Reconhecimento dos componentes; 5 Montagem mecânica; 6 - Montagem eletro/eletrônica; 7 - Testes preliminares do protótipo.

\subsection{Etapa 4 - Reconhecimento dos componentes}

Nesta etapa o aluno deve manipular cada um dos componentes do sistema físico a ser montado. Espera-se também que o estudante ou grupo especifique cada um dos componentes, fazendo um levantamento objetivo de cada uma das peças que compõem o protótipo a ser construído. Do ponto de vista físico, as principais partes a serem reconhecidas e especificadas, como mostrado na Figura 2, são a plataforma física (a), os sensores (b), os motores (c) e as placas eletrônicas (d). 
Figura 2 - Principais componentes a serem especificados.

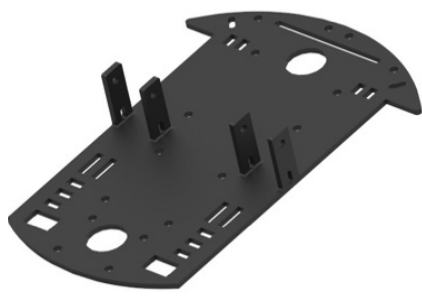

(a)

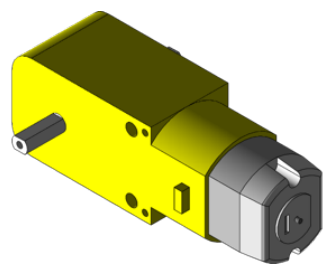

(c)

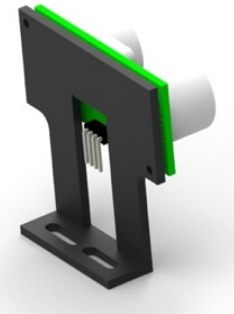

(b)

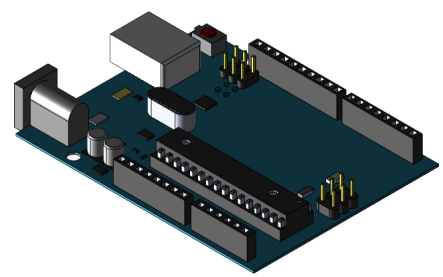

(d)

Fonte: https://grabcad.com/library

\subsection{Etapa 5 - Montagem mecânica}

Uma vez entendidas a funcionalidade e aplicabilidade de cada componente, na etapa anterior, deve-se buscar uma forma de se distribuir cada um desses elementos no espaço, de acordo com sua funcionalidade.

Aqui procura-se uma visualização mais clara da montagem dos componentes, e então o aluno desenvolve habilidades tanto manuais quanto estéticas. Deve-se procurar alocar os componentes na plataforma (chassi), da forma mais eficiente possível, sempre atentando para os requisitos mecânicos como centro de gravidade e estabilidade do conjunto.

A capacidade de elaboração e de trabalho em equipe toma dimensões importantes, uma vez que os membros de um mesmo grupo devem aprender a comunicar suas ideias $e$ a escutar o colega, na busca de uma solução para a equipe. Estas habilidades de trabalho em grupo, que se manifestam a partir desta etapa, serão cada vez mais exploradas ao longo do projeto de forma a que se tenha um crescimento dos soft skills necessários ao bom desenvolvimento de cada integrante e ao sucesso da equipe.

\subsection{Etapa 6 - Montagem eletro/eletrônica}

O aluno precisa apreender que o robô é incapaz de se mover sozinho, acionar os motores ou fazer a leitura dos sensores. Então, esses precisam ser conectados eletricamente. Isso significa que é preciso entender a necessidade elétrica desses componentes, como tensão, corrente elétrica e o sentido que essa corrente percorrerá no circuito conectado.

Aqui, novas habilidades são exigidas e novas competências, adquiridas. O conhecimento de física eletromagnética e de eletricidade básica surge como necessidade para se compreender o problema do ponto de vista eletro/eletrônico. Notadamente, a questão da diferença entre tensão e potência se torna evidente e o aluno deve perceber 


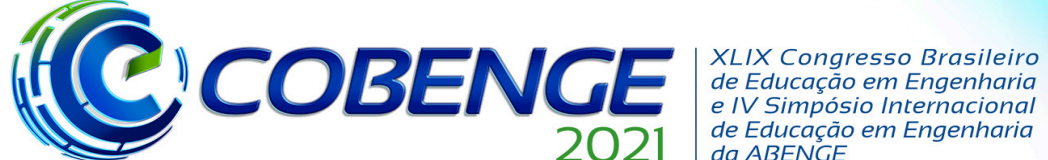 28 a 30 de SETEMBRO

que, para se acionar um motor elétrico, é exigida potência elétrica, incapaz de ser obtida diretamente das saídas de baixa corrente do Arduino.

Para que os motores possam ser comandados pelo microprocessador, é necessário que haja uma fonte externa capaz de fornecer potência aos mesmos. Esta potência deverá ser fornecida por algum elemento como um transistor de potência, como se vê na Figura 3 (a) ou por uma ponte $\mathrm{H}$, como se pode ver na Figura 3 (b), a qual permite o controle do sentido de rotação, fazendo com que o carro possa ir para frente ou para trás.

Figura 3 - Circuitos de potência para controle dos motores.
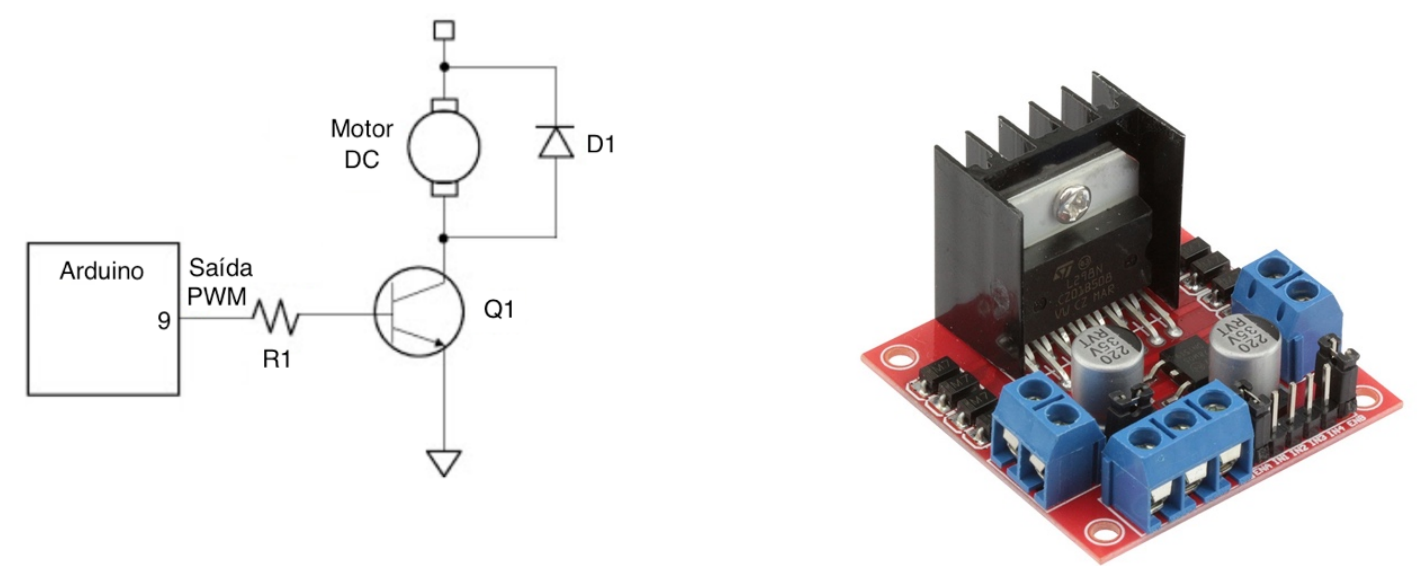

(a) Transistor de potência

(b) Ponte $\mathrm{H}$

Fonte: https://blog.eletrogate.com/guia-definitivo-de-uso-da-ponte-h-I298n/

No mesmo caminho de entendimento, deve ficar claro que os sensores, por sua vez, já que são dispositivos de baixa potência, podem ser conectados diretamente às entradas do Arduino.

\subsection{Etapa 7 - Testes preliminares do protótipo}

Cumpridas as etapas anteriores, tem-se como resultado uma montagem típica do carro robótico, como ilustra a Figura 4, em que se veem completadas tanto a montagem mecânica quanto a montagem eletro/eletrônica.

Figura 4 - Montagem final típica do carro robótico.

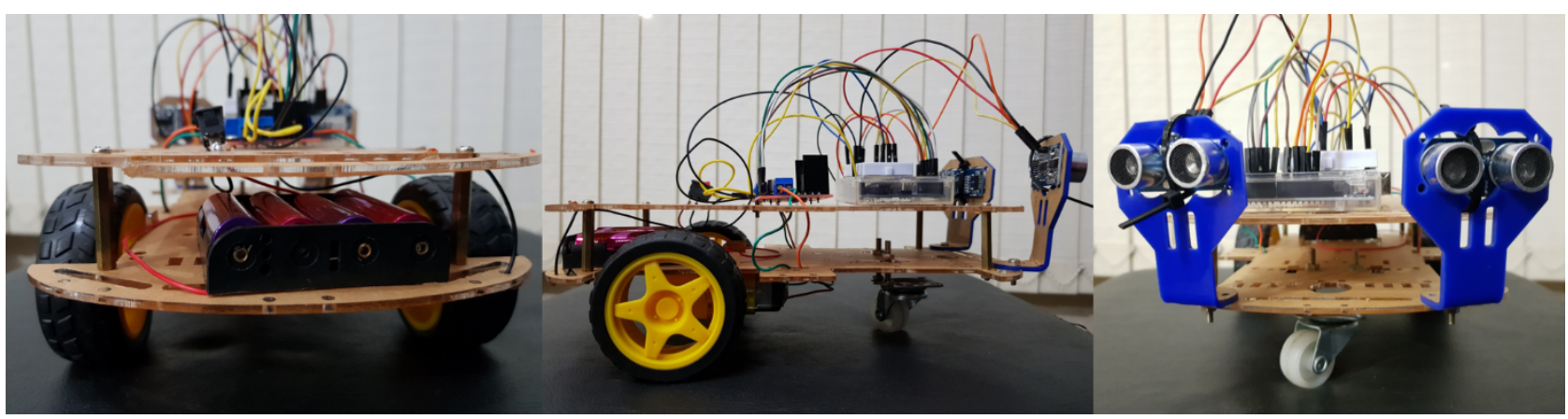

Fonte: Os autores

Primeiramente, cada um dos componentes deve ser testado e seu funcionamento comprovado. Aqui se tem, portanto, uma janela de oportunidade para que se esclareçam as dúvidas que surgiram no processo de montagem do carro robótico, de forma a que o grupo, como um todo único e coeso, possa prosseguir. Portanto, os integrantes do grupo 


\section{(C) COBENCE

irão interagir para que aquele que tenha compreendido certo aspecto possa esclarecer aos demais a operação ou necessidade de algum componente ou funcionalidade.

Ao se desenvolverem os testes preliminares sobre o protótipo físico, ao mesmo tempo em que se estabelece o correto funcionamento do mesmo, reparando o modelo, quando necessário, promove-se uma maior interação dos integrantes do grupo e o estabelecimento de uma troca de informações entre eles.

Ao final desta etapa, espera-se um protótipo totalmente funcional e destituído de bugs, que esteja apto a ser integrado ao seu gêmeo digital. Mais do que isto, esta etapa serve para se reforçarem as inter-relações entre os componentes do grupo, fortalecendo o aspecto de trabalho em equipe, de forma multidisciplinar e colaborativa.

\section{FASE VIRTUAL (III)}

A fase Virtual é aquela em que o grupo tem contato direto com o software, os elementos lógicos e as rotinas de programação, tanto dos componentes visuais quanto da comunicação de dados entre os gêmeos.

Esta Fase possui três Etapas: 8 - Reconhecimento da plataforma virtual; 9 Construção do modelo virtual; 10 - Programação da interatividade.

\subsection{Etapa 8 - Reconhecimento da plataforma virtual}

$\mathrm{Na}$ etapa de reconhecimento da plataforma virtual, o grupo deve interagir com as ferramentas de software que serão utilizadas, tanto na programação das ações do carro robótico físico, quanto no desenvolvimento do modelo virtual e suas posteriores programações de comportamento e comunicação.

Nesta etapa os alunos devem lidar com as ferramentas de software, desde a instalação até o uso das mesmas. É uma etapa de familiarização com o ambiente virtual que eles deverão utilizar para o desenvolvimento do gêmeo digital e da programação do carro robótico físico.

\subsection{Etapa 9 - Construção do modelo virtual}

A construção do modelo virtual é o momento em que o grupo irá utilizar a aplicação CoppeliaSim (COPPELIA, 2021) para construir um modelo gráfico e funcional do carro robótico, em ambiente virtual.

Este ambiente posiciona um modelo 3D de um objeto complexo em um espaço virtual e é capaz de movimentá-lo neste espaço.

Aqui deverão ser aprendidas e utilizadas habilidades de projeto tanto no plano quanto no espaço, para que se componham as partes do todo do robô, conforme ilustra a Figura 5.

A escolha do software em questão se dá tanto pela grande capacidade de projeto e representação gráfica do mesmo, quanto pelo fato de que esta aplicação é de uso livre para fins não comerciais. Desta forma, os alunos podem ter acesso ao programa, instando-o em suas próprias máquinas, o que facilita a integração do trabalho pelo grupo. 


\section{COBENGE de Educação em Engenharia
da ABENGE \\ 28 a 30 de SETEMBRO}

Figura 5 - Gêmeo virtual no software Coppelia.

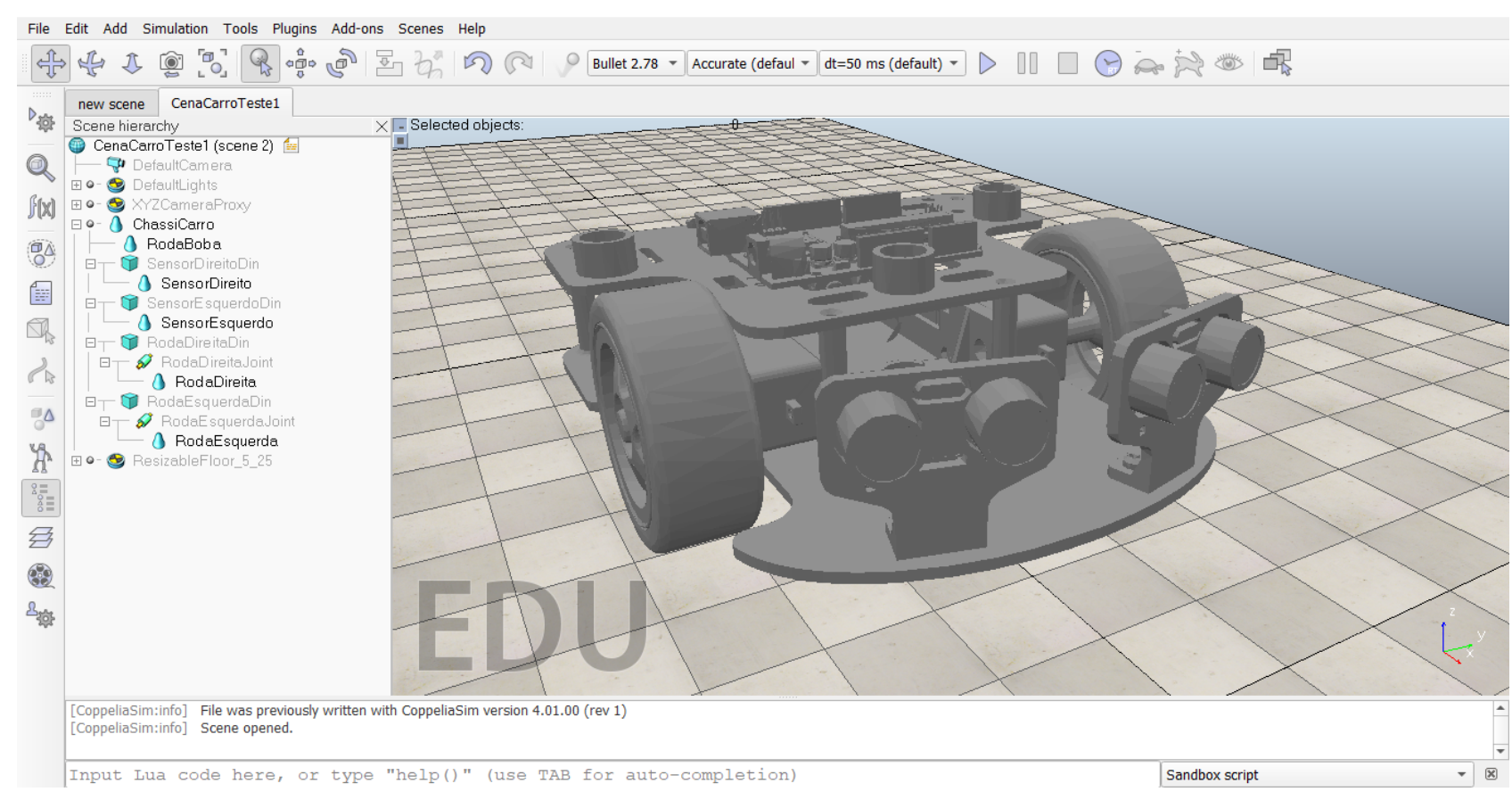

Fonte: Os autores

\subsection{Etapa 10 - Programação da interatividade}

A programação da interatividade é a fase final do desenvolvimento do projeto, em que as partes, tanto física quanto virtual precisam interagir. Para tanto, há que se desenvolver códigos de programação em ambos lados do sistema.

Estes códigos envolvem a criação de um padrão de comunicação entre o microcomputador, que está executando o ambiente virtual CoppeliaSim e o Arduino, que controla o carro robótico. Este padrão é, portanto, um protocolo de comunicação entre estas duas entidades. Neste sentido, o desafio de se compreender tanto a questão de alto nível da comunicação entre as partes e o estabelecimento de um mecanismo de troca de informações entre elas, quanto a questão prática e funcional de se programar uma sequência de códigos de instruções, que deve operar de forma sincronizada entre dois ambientes distintos, se apresentam.

Do ponto de vista do protótipo físico, há a necessidade de programação do Arduino para que ele possa tanto receber quanto enviar dados ao microcomputador, que está executando o gêmeo virtual. Isto é possível, na prática, através do emprego de funções de comunicação de dados pela porta serial do Arduino.

Devem-se estabelecer, portanto, funções de tratamento de strings para que se possam interpretar os comandos recebidos através da porta serial e proceder ao correto deslocamento do carro robótico, comandando-se os motores do mesmo, como ilustram as funções implementadas, movAvante() e movCurvaEsquerda(), no código da Figura 6 (a).

Por outro lado, o gêmeo digital implementado no CoppeliaSim também deve enviar e receber dados do seu simétrico físico. Isso se dá, conforme visto na Figura 6 (b), através das funções SerialSend e SerialRead e do posterior tratamento das strings ali enviadas e recebidas. $\mathrm{O}$ código para envio e recebimento de mensagens entre o CoppeliaSim e o Arduino foi adaptado de Eric Rohmer (ROHMER, 2004). 
Figura 6 - Fração de código para comunicação de dados.

$\ldots$

void $\operatorname{loop}()\{$

dist1 $=$ sensor 1()$; / /$ leitura de distancia do sensor 1
dist2 $=$ sensor 2()$; / /$ leitura de distancia do sensor 2

Serial.print(dist1);

Serial.print(","); //separador de dados

Serial.println(dist2); //finaliza a string que é passada para o Coppelia, sendo o valor "dist2\n"

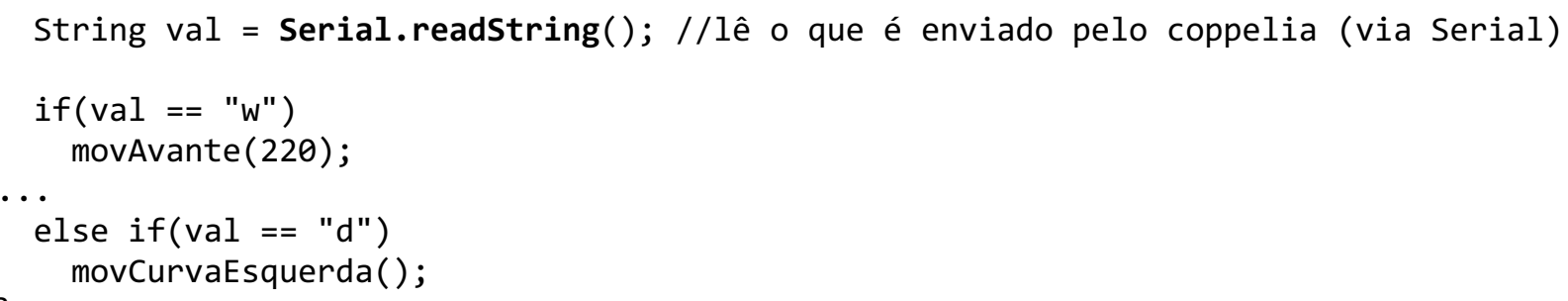

(a) Comunicação de dados pelo Arduino.

-- This code is provided by Eric Rohmer

- - erohmer@gmail.com

-- you can use it without any warranty and modify reuse and distribute it at will

...

serial=sim.serialopen (portNumber, baudrate)

...

--read a full line

str=sim. serialRead (serial, 100, true, ' $\backslash n$ ' , 100)

...

-- Codigo para ler mensagem e comandos do teclado

...

sim.serialsend(serial, "w") - - frente

elseif(auxiliaryData[1]==string.byte('s')) then

sim.serialsend(serial,"s") -- tras

elseif(auxiliaryData[1] ==string.byte('a')) then

...

elseif(auxiliaryData[1]==string.byte('d')) then

...

sim.serialclose(serial)

(b) Comunicação de dados pelo CoppeliaSim

Fonte: Os autores

\section{CONCLUSÃo}

Além das habilidades desenvolvidas em cada etapa, para concluir o projeto como um todo, os alunos são instigados, ainda, ao raciocínio lógico, as relações pessoais, à integração de conceitos aprendidos para o desenvolvimento de projetos, à utilização da criatividade em diferentes situações, e à capacidade crítica. 


\section{COBENCE

Ainda, a oportunidade de vivenciar a experiência de um projeto aplicado exercita a busca pelo conhecimento aprofundado, sendo um exemplo a ser seguido ao longo do curso.

A partir do conjunto de habilidades desenvolvidas, somado às capacidades de eletrônica, mecânica e programação adquiridas, os alunos começam a desenvolver também as competências interdisciplinares e interpessoais, necessárias à formação em engenharia.

Essas habilidades, desenvolvidas durante o projeto, compõem um arcabouço de competências tanto em soft skills quanto em hard skills, tidas, atualmente, como algo desejável e a se alcançar.

\section{REFERÊNCIAS}

ARDUINO. Site oficial. Disponível em: <http://arduino.cc>. Acesso em: 23 de abril de 2021.

COPPELIA. Site oficial. Disponível em: < https://coppeliarobotics.com/downloads>. Acesso em: 23 de abril de 2021.

CORDON, Heloisa C. F.; NETO, Octavio M. Aplicação do Team Based Learning nas aulas de laboratório de materiais de construção civil num curso de engenharia. In: XLVIII Congresso Brasileiro de Educação em Engenharia, 2020, Evento On Line. Anais. Disponível em: http://abenge.org.br/sis_artigo_doi.php?e=COBENGE\&a=20\&c=3041. Acesso em: 20 março.2021.

LUCA, C. D. Gêmeos Digitais vão impulsionar a Transformação Digital. Disponível em: $<$ https://cio.com.br/em-2019-gemeos-digitais-vao-impulsionar-a-transformacao-digital/> Acesso em 15 mar. 2020.

Ministério da Educação. Resolução CNE/CES n 2, de 24 de abril de 2019, Diretrizes Curriculares Nacionais do Curso de Graduação em Engenharia.

Disponível em: <http://portal.mec.gov.br/escola-de-gestores-da-educacao-basica/323secretarias-112877938/orgaos-vinculados-82187207/12991-diretrizes-curriculares-cursosde-graduacao>

PIROLA, Nelson A. org. Ensino de ciências e matemática. Vol. IV. São Paulo: Editora UNESP; São Paulo: Cultura Acadêmica, 2010. E-book.

ROHMER, E. Arduino + compass+ PSD sensor +V-REP simulation. Disponível em: https://www.youtube.com/watch?v=ZJQV2FIGFBo. Acesso em: 10 Fev. 2021.

\section{ROBOTICS AS A METHODOLOGY IN SUPPORT OF THE INTRODUCTION TO ENGINEERING CLASS}

Abstract: A teaching support methodology is presented, to be used in introduction to engineering class or similars, which uses elements of robotics and proposes the 
development of both a physical and virtual prototype. It takes advantage of the concept of virtual twins to introduce the student to engineering, according to the current DCNs (National Curriculum Guidelines) of the Undergraduate Engineering Course, to develop both soft and hard skills as expected of an engineer. It is proposed, through a series of learning activities, to explore theoretical and practical aspects of the construction of a robotic prototype and its virtual counterpart, with the help of free software.

Keywords: Methodology. Robotics. Introduction to engineering class. TBL. 